\title{
Topical ALA-Photodynamic Therapy Combined with Acne Debridement and Meticulous Nursing for the Treatment of Moderate-Severe Acne in Adolescent Patients
}

\section{Rongxin Ren \\ Shiwei Bao \\ Wenjiang Qian \\ Hongyi Zhao}

Department of Plastic Surgery, Beijing Hospital, National Center of Gerontology, Institute of Geriatric Medicine, Chinese Academy of Medical Sciences, Beijing, People's Republic of China
Correspondence: Hongyi Zhao Email zhaohongyi99I@yeah.net
Objective: The present study aims to explore the effect of acne debridement + meticulous nursing on 5-aminolevulinic acid photodynamic therapy (ALA-PDT) in adolescent patients with moderate-severe acne.

Methods: A total of 60 adolescent patients with moderate-severe acne who were admitted to our plastic surgery outpatient clinic between January 2018 and January 2020 were selected as the subjects of the present retrospective study. The patients were divided into two groups: the observation group and the control group $(n=30$, each). Patients in the control group were treated with standardized ALA-PDT and conventional nursing, while patients in the observation group were treated with ALA-PDT and acne debridement + meticulous nursing intervention. The treatment's therapeutic effect, adverse reaction incidence, and patient satisfaction 6 months after treatment were compared between the two groups.

Results: The acne debridement + meticulous nursing effectiveness was $86.7 \%$ in the observation group and $60 \%$ in the control group, and the adverse reaction incidence was $20 \%$ in the observation group and $46.7 \%$ in the control group. There existed statistically significant differences in the above-stated indicators between the two groups $(p<0.05)$. The difference in the visual analogue scores (VASs) for pain, which were measured immediately after the operations, between the two groups was not statistically significant $(\mathrm{p}>0.05)$; however, the respective VAS differences between the two groups at $30 \mathrm{~min}$ and $60 \mathrm{~min}$ after the operation were statistically significant $(\mathrm{p}<0.05)$. There was a statistically significant difference in patient satisfaction between the two groups $(\mathrm{p}<0.05)$.

Conclusion: In adolescent patients with ALA-PDT-treated moderate-severe acne, the application of acne debridement + meticulous nursing could improve the clinical treatment efficacy and patient comfort as well as reduce the adverse reaction incidence. For these reasons, the treatment method could be worth promoting in clinical practice.

Keywords: photodynamics, 5-aminolevulinic acid, psychology, pain, meticulous nursing, acne debridement

Acne is a chronic inflammatory skin disease that often occurs in adolescent patients (adolescence is a specific period in which individuals undergo rapid development as well as physical and psychological changes). It most commonly appears on the face, chest, and back. Acne types can be divided into mild, moderate, and severe acne according to the disease's extremity. Due to its large skin lesion area, severe acne can be destructive to the patient's appearance. If it is not diagnosed and treated 
early, it can also make serious psychological burdens for adolescent patients, affecting their compliance and the treatment's effects. This will not only affect the patient's quality of life but also lead to varying degrees of family and social problems. ${ }^{1}$ In recent years, accumulated literatures have confirmed 5-aminolevulinic acid-photodynamic therapy (ALA-PDT), which is based on metabolites produced by hair follicle sebaceous glands after the topical application of 5-aminolevulinic acid, as an effective new acne treatment method. Following irradiation by a certain light source, ALA-PDT generates active oxygen, killing the Propionibacterium acnes and destroying the hair follicle sebaceous glands. ${ }^{2,3}$ Another key point is to increase the therapeutic adherence for acne patients in order to reduce side effects such as erythema, dryness and desquamation. ${ }^{4}$ However, the ssstreatment is correlated with significant intraoperative pain and postoperative adverse reactions, such as erythema, hyperpigmentation, and desquamation, all of which have serious psychological impacts on adolescent patients. There is currently a lack of experience regarding meticulous clinical nursing in adolescent patients. It has been found by $\mathrm{Wu}$ et $\mathrm{al}^{5}$ that photodynamic therapy combined with acne squeezing is effective in the treatment of moderate-severe acne; it also reduces the incidence of recurrence. In the present study, acne debridement + meticulous nursing interventions were applied. This intervention includes the treatment and care of preoperative (acne debridement pretreatment), intraoperative (nursing during treatment) and postoperative (skin nursing, psychological care, daily nursing skincare product application, and lifestyle and diet education). This intervention carries out comprehensive nursing from the acne part, psychology and physiology of patients, which aims to treat adolescent patients with moderate-severe acne, and relatively satisfactory results were achieved. The details are reported as follows.

\section{Subjects and Methods}

\section{Study Subjects}

A total of 60 adolescent patients (54 females and 6 males) with moderate-severe acne who were admitted to our hospital between January 2018 and January 2020 were selected as the study subjects. The patients were divided into two groups, the control group and the observation group $(n=30$, each), using retrospective analysis of treatment and nursing methods. The inclusion criteria referred to the diagnosis of Grade III-IV acne in accordance with the Pillsbury scale. The exclusion criteria were: (1) patients treated with antibiotics within 1 month before treatment; (2) patients who took retinoids within the 6 months before treatment; (3) patients with other skin diseases; and (4) patients with a history of photosensitivity or keloids. The patients were aged 15-20 years, with an average age of 16 years. The minimum disease duration was 6 months, and the maximum duration was 36 months, with an average duration of 10.8 months. Clinical manifestations of the disease included multiple inflammatory lesions (eg, papules, pustules, cysts, and nodules) on the face (Table 1). This study was conducted with approval from the Ethics Committee of Beijing Hospital (No: 2021BJYYEC-163-01). Written informed consent was obtained from all participants.

\section{Intervention Methods}

\section{The Control Group}

After routine facial cleansing, 30 patients were treated with standardized ALA-PDT. The therapeutic measures were as follows: $10 \%$ of the ALA gel solution (produced by Shanghai Fudan Zhangjiang Biomedical Co., Ltd.) was applied evenly onto the lesion and in a $0.5-1.0 \mathrm{~cm}$ range of the surrounding area. The local area was closed with a bandage for $60 \mathrm{~min}$ to prevent contact with light. After washing the face using sterile normal saline, a lightemitting diode photodynamic therapy instrument (produced by Wuhan YAG Photoelectric Technology Co.,

Table I General Characteristic Comparison Between the Two Groups (x $\pm s)$, (n), and [n (\%)]

\begin{tabular}{|c|c|c|c|c|c|c|c|c|c|}
\hline \multirow[t]{2}{*}{ Groups } & \multirow[t]{2}{*}{ Cases } & \multirow[t]{2}{*}{ Age } & \multicolumn{2}{|c|}{ Gender } & \multicolumn{3}{|c|}{ Types of Lesions } & \multicolumn{2}{|c|}{ Course of Disease } \\
\hline & & & $\mathbf{M}$ & $\mathbf{F}$ & Papules & Pustules & Cysts & $>$ I Year & $<$ Year \\
\hline Observation group & 30 & $16.40 \pm 1.28$ & 3 & 27 & $\mathrm{II}$ & 8 & 2 & 18 & 12 \\
\hline Control group & 30 & $16.60 \pm 1.33$ & 2 & 28 & 10 & 7 & 3 & 16 & 14 \\
\hline$\chi^{2} / \mathrm{t}$ & & 0.595 & \multicolumn{2}{|c|}{0.218} & \multicolumn{3}{|c|}{0.290} & \multicolumn{2}{|c|}{0.271} \\
\hline$P$ & & 0.554 & \multicolumn{2}{|c|}{0.640} & \multicolumn{3}{|c|}{0.865} & \multicolumn{2}{|c|}{0.602} \\
\hline
\end{tabular}


Ltd.) was used for $633 \mathrm{~nm}$ wavelength irradiation, with an output power of $20 \mathrm{~mW} / \mathrm{cm}^{2}$, average irradiation dose of $80 \mathrm{~J} / \mathrm{cm}^{2}$, spot area of $15 \mathrm{~mm} \times 40 \mathrm{~mm}$, pulse width of 30 $50 \mathrm{~ms}$, and distance of $10-20 \mathrm{~cm}$ from the face. The patient's eyes were protected with light-shielding glasses during the irradiation. The duration of each treatment was 20 min once a week, and the duration of one treatment course was 4 weeks. The patients were followed up with until 6 months after the treatment.

\section{The Observation Group}

A total of 30 patients were pretreated with acne debridement in a sterile operating room. Next, they were treated with standardized ALA-PDT therapy (the same as in the control group) combined with meticulous nursing. The specific operational measures were as follows:

(1) Acne debridement pretreatment: After face cleansing, the ionic spray machine was used for 10 minutes. Next, the face treatment area was disinfected with iodophor, and the acne lesions, including pimples, papules, pustules, and cysts, were pretreated. Pustules and pimples were punctured using a needle, and pustules were squeezed to remove the pus. During the operation, mechanical injury caused by excessive force, which increases the risk of acne scarring, should be avoided, and close attention should be paid to the aseptic operation principle in order to avoid skin infection. After completing the debridement of all facial lesions, the treatment area was again disinfected with iodophor. ${ }^{6}$

(2) Nursing during treatment: When applying the gels containing ALA, sensitive areas, such as the lips and eyelids, should be avoided. If a small amount of liquid is inadvertently applied to the mucosal area, the photosensitizer must be removed thoroughly after bandaging in order to reduce residue as well as avoid postoperative redness and pain. ${ }^{7}$

(3) Postoperative skin nursing: Immediate postoperative skin reactions include erythema and swelling. In the present study, medical cold compresses containing hyaluronic acid or antibiotic peptides were applied to the patients' faces and intermittently ice-packed for 30-60 min using a cold spray machine. The cold compresses were removed after the redness and swelling subsided. Then, 2-3 patches were applied daily for one week to promote skin barrier repair. If skin desquamation occurred after the erythema faded, the patient was advised to apply a cream containing hyaluronic acid dressing 2-3 times a day to enhance facial moisturization and facilitate skin barrier repair. ${ }^{8}$
(4) Pain care: Before the treatment, patients were questioned about their usual pain tolerance; in the case of pain intolerance, communication with the doctor about analgesic medication before the treatment was necessary. ${ }^{9}$

(5) Psychological care: Psychological interventions for adolescent patients was individualized; this intervention was conducted in a relatively quiet and undisturbed treatment room before each treatment, as excessive disturbance by other patients can cause anxiety. Additionally, simple language or popular science terms was adopted when introducing the pathogenesis and treatment in order to avoid textbook communication. Furthermore, questions raised by the patient were explained calmly, negative patient emotions correctly identified, and anxiety levels assessed in time. Special attention was paid to protecting patient privacy. Moreover, one was also careful not to hurt the patient's self-esteem with wording, and family members should be instructed to care about the psychological problems of the adolescent girls and appropriately provide them with encouragement and support. ${ }^{10}$

(6) Daily nursing skincare product application: The patients included in the present study were instructed to choose appropriate skincare products according to their skin type and lesions. In patients who mainly had acne and pimples, along with generally oily skin, skincare products containing oilcontrolling, keratolytic, exfoliating, anti-inflammatory, and skin-barrier-repairing active ingredients were adopted for daily care. The skincare process comprised: (1) cleansing with a neutral or weak alkaline cleanser; (2) applying an oil control and skin barrier repair gel; and (3) applying a lotion with keratolytic and anti-inflammatory effects on local lesions. Skin nursing was conducted twice a day. Attention was paid to applying warm water, washing the face with gentle movements, and avoiding the use of any exfoliating bath products or facial cleansers that damage the skin barrier. The patients were instructed to observe the skin lesions over time after application in order to reduce skin allergies caused by improper skincare product use, conduct skincare patch tests if necessary, and discontinue the application immediately if allergic reactions occurred during the application. In the case of severe skin desquamation or itching in the treated area, patients were advised to use medical skincare products under medical supervision. ${ }^{9,11}$

(7) Lifestyle and diet education: The patients were assisted in preparing a science-supported and healthy diet according to their lifestyle and current diet. The patients were advised to reduce milk intake in their daily lives. ${ }^{12}$ 
Table 2 Therapeutic Effect Comparison Between the Two Groups

\begin{tabular}{|l|c|c|c|c|c|c|c|}
\hline Groups & Cases & Recovery & Markedly Effective & Effective & Invalid & \multicolumn{2}{c|}{ Total Treatment Effectiveness } \\
\cline { 3 - 7 } & & & & & & Cases & 26 \\
\hline Observation group & 30 & 22 & 2 & 2 & 4 & 12 & 18 \\
\hline Control group & 30 & 13 & 1 & 4 & 60 \\
\hline
\end{tabular}

\section{Observation Indicators}

(1) Therapeutic effect judgment: The lesion evaluation was based on the lesion remission rate ${ }^{13}$ ([number of lesions before treatment - number of lesions after treatment])/number of lesions before treatment $\times 100 \%$ ), and was divided into four grades: basic healing, significantly effective healing, effective healing, and ineffective healing. Basic healing was defined as a $\geq 90 \%$ lesion remission, significantly effective healing as a $60-89 \%$ lesion remission, effective healing as a $20-59 \%$ lesion remission, and ineffective healing as a $<20$ lesion remission. The total therapeutic effectiveness $=$ (cases with basic healing + cases with significantly effective healing)/total cases $\times 100 \%$.

(2) Adverse reaction incidence: The incidence of erythema, edema, desquamation, and acne scarring, which commonly occurred during treatment.

(3) Pain scoring: The visual analogue score (VAS) ranged from 0-10 points, with $0-1$ point meaning no pain, 2-3 points meaning mild pain, $4-5$ points meaning moderate pain, and $>5$ points meaning severe pain. The score was determined by the patients themselves according to their pain conditions.

(4) Patient satisfaction evaluation: our hospital satisfaction survey was adopted to determine patient satisfaction. The survey was divided into three items: very satisfied, satisfied, and dissatisfied. The satisfaction rate $=$ (number of very satisfied cases + number of satisfied cases)/total number of cases $\times 100 \%$.

\section{Statistic Analysis}

The Statistical Package for Social Sciences 20.0 software was adopted for statistical data analysis. The measurement data were expressed as mean \pm standard deviation $(x \pm s)$; the $t$-test was used for comparisons between the two groups. The countable data were expressed in either frequency or percentage (\%); the $\chi^{2}$ test or the Fisher exact probability method was adopted for comparisons between the two groups. A $\mathrm{p}$ value of $<0.05$ was considered statistically significant.

\section{Results}

\section{Effectiveness Comparison Between the Two Groups}

The total treatment effectiveness was $86.7 \%$ in the observation group and $60 \%$ in the control group $\left(\chi^{2}=5.455\right)$; the difference between the two groups was statistically significant $(p<0.05)($ Table 2, -4). Written informed consent has

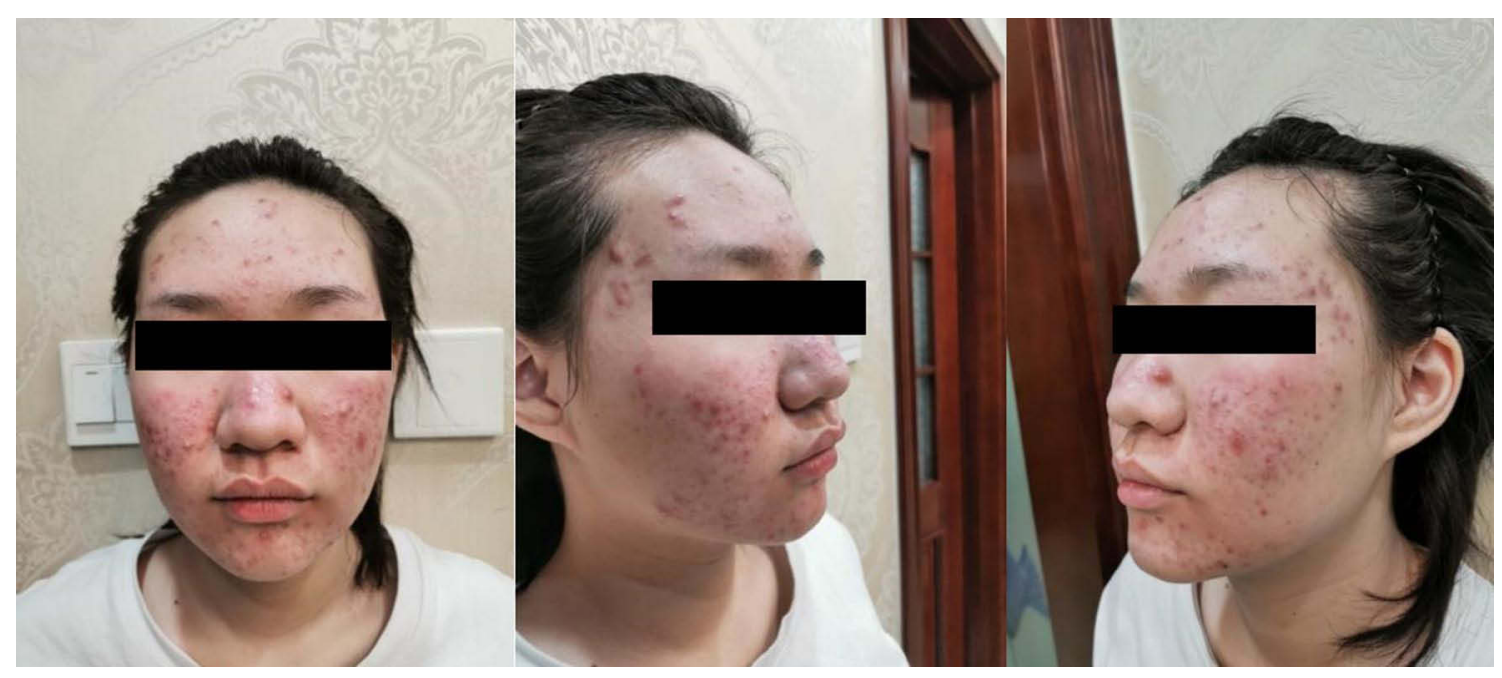

Figure I A female patient in control group: before treatment. 


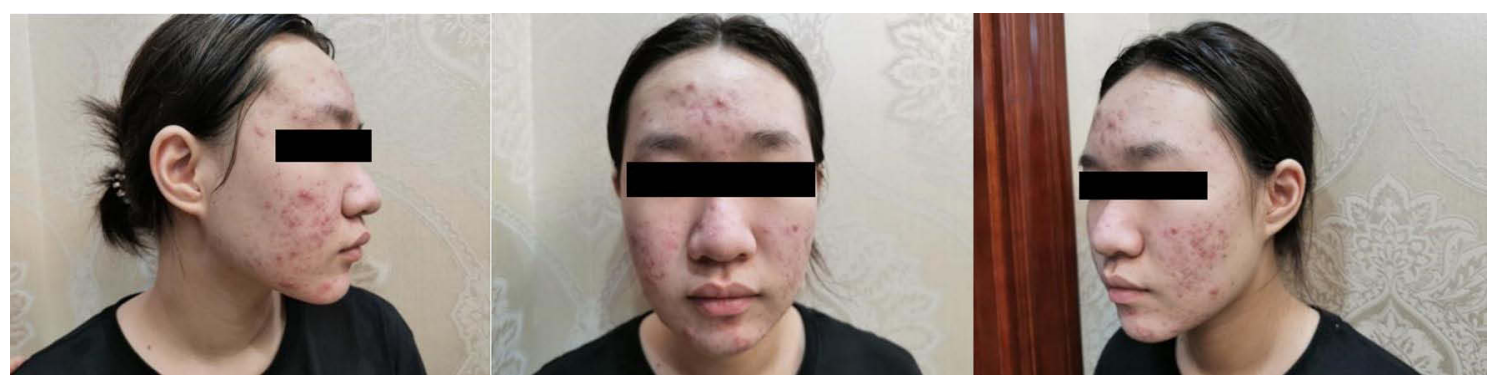

Figure $2 \mathrm{~A}$ female patient in control group: follow up 6 months after treatment.

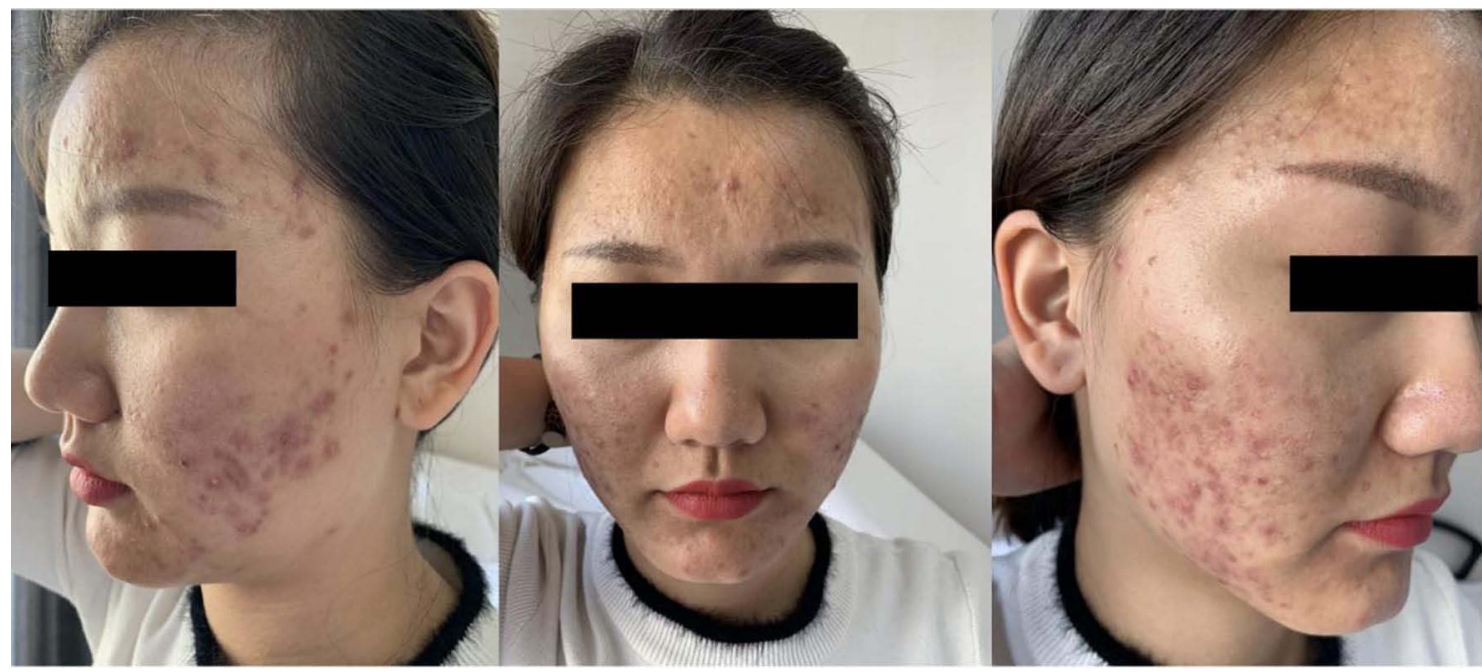

Figure 3 A female patient aged 20 of the observed group. Frontal and bilateral clinical photos of the face before treatment.

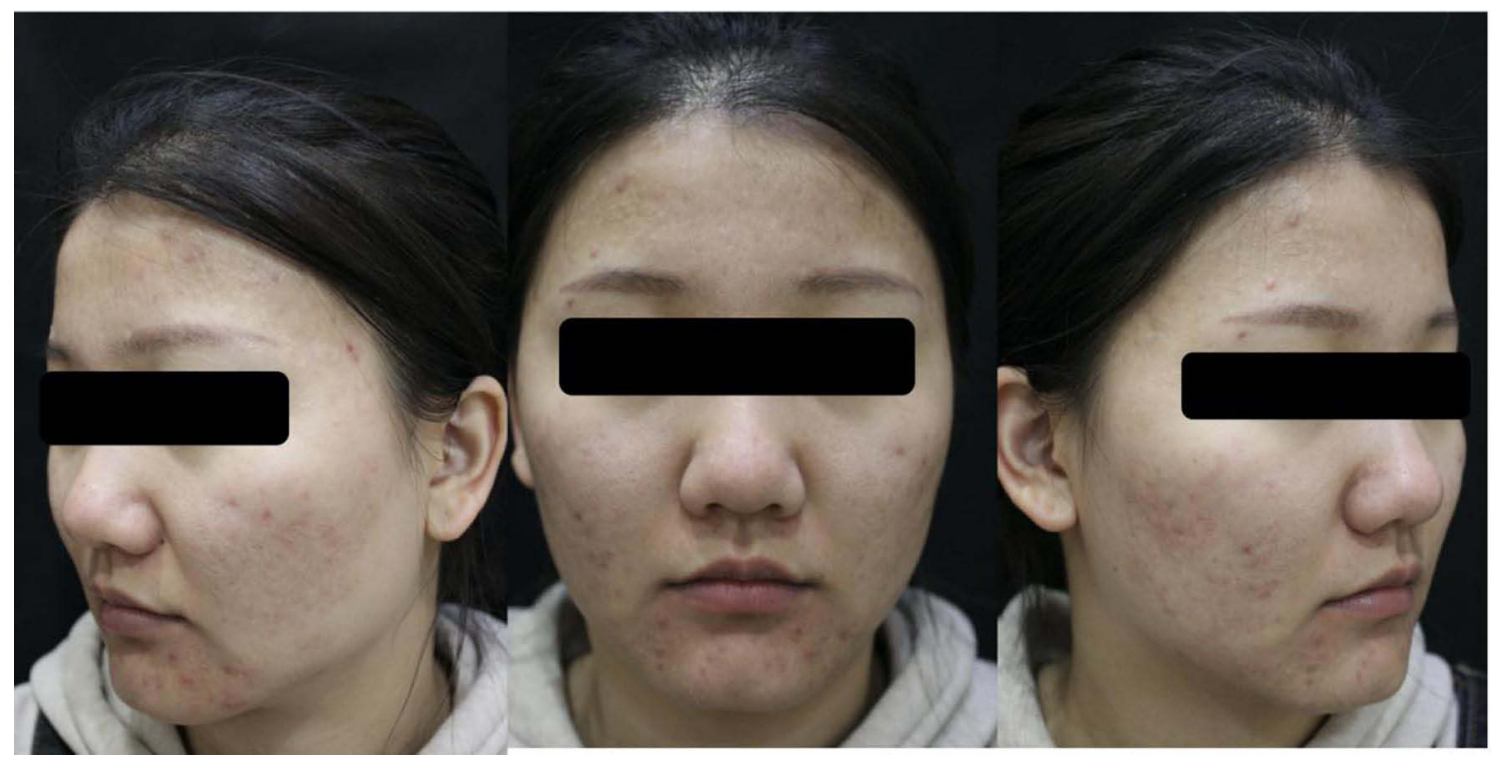

Figure $4 \mathrm{~A}$ female patient aged 20 of the observed group. Frontal and bilateral clinical photos of the face 6 months after treatment. 
Table 3 Adverse Reaction Incidence Comparison Between the Two Groups Three Days After Treatment

\begin{tabular}{|l|c|c|c|c|c|c|c|c|c|}
\hline \multirow{2}{*}{ Groups } & \multirow{2}{*}{ Cases } & \multicolumn{2}{|c|}{ Erythema } & \multicolumn{2}{|c|}{ Swelling } & \multicolumn{2}{|c|}{ Itching } & \multicolumn{2}{c|}{ Total } \\
\cline { 3 - 10 } & & Cases & $\%$ & Cases & $\%$ & Cases & $\%$ & Cases & $\%$ \\
\hline Observation group & 30 & 0 & 0 & 1 & 3.3 & 5 & 16.7 & 6 & 20 \\
\hline Control group & 30 & 5 & 16.7 & 5 & 16.7 & 7 & 23.3 & 17 & 56.7 \\
\hline
\end{tabular}

been provided by the patient to have the case details and any accompanying images published.

\section{Adverse Reaction Incidence Comparison Between the Two Groups}

Patients in both groups experienced different degrees of erythema, swelling, pain, and other adverse reactions immediately after the operation. The reactions improved with symptomatic treatment; however, the relief came more slowly in the control group than in the observation group. The respective incidences of erythema, swelling, and itching after three days of treatment were compared between the two groups $\left(\chi^{2}=8.531\right)$; the difference was statistically significant $(\mathrm{p}<0.05$ in all) (Table 3$)$.

\section{Postoperative VAS Comparison Between the Two Groups}

There was no statistically significant difference in the VAS between the two groups immediately after treatment; however, the VAS in the observation group decreased significantly at $30 \mathrm{~min}$ and $60 \mathrm{~min}$ after the operation, respectively; the differences between the two groups were statistically significant ( $<0.05$ in both) (Table 4 ).

\section{Postoperative Patient Satisfaction Comparison Between the Two Groups}

The patient satisfaction was $90 \%$ in the observation group and $73.3 \%$ in the control group $\left(\chi^{2}=4.812\right)$; the difference was statistically significant $(\mathrm{p}<0.05)$ (Table 5).

\section{Discussion}

Acne is a dermatological disease commonly occurring in adolescent girls. The disease pathogenesis is complex, and it is correlated with excessive sebum production, abnormal keratinization of follicular sebaceous gland openings, the P. acnes infection, androgens, genetics. ${ }^{14}$ Currently, the treatment methods mainly include oral medication and topical treatments. However, the adverse effects and drug resistance of oral medication are the main worrying issues for adolescent patients and their parents. In the new

Table 4 Postoperative VAS Comparison Between the Two Groups

\begin{tabular}{|l|c|c|c|c|}
\hline \multirow{2}{*}{ Groups } & \multirow{2}{*}{ Cases } & \multicolumn{3}{|c|}{ VAS } \\
\cline { 3 - 5 } & & Immediately After Operation & $\mathbf{3 0}$ min After the Operation & $\mathbf{6 0}$ min After the Operation \\
\hline Observation group & 30 & $7.90 \pm 0.80$ & $4.40 \pm 0.50$ & $1.43 \pm 0.50$ \\
\hline Control group & 30 & $7.83 \pm 0.75$ & $6.50 \pm 0.51$ & $3.47 \pm 0.51$ \\
\hline$\chi^{2 / t}$ & & 0.333 & 16.155 & 15.572 \\
\hline$P$ & & 0.740 & $<0.01$ & $<0.01$ \\
\hline
\end{tabular}

Table 5 Patient Satisfaction Comparison Between the Two Groups

\begin{tabular}{|l|c|c|c|c|c|c|c|c|c|}
\hline \multirow{2}{*}{ Groups } & \multirow{2}{*}{ Cases } & \multicolumn{2}{|c|}{ Very Satisfied } & \multicolumn{2}{|c|}{ Satisfied } & \multicolumn{2}{c|}{ Dissatisfied } & \multicolumn{2}{c|}{ Satisfaction } \\
\cline { 3 - 10 } & & Cases & $\%$ & Cases & $\%$ & Cases & $\%$ & Cases & $\%$ \\
\hline Observation group & 30 & 25 & 83.3 & 2 & 6.7 & 3 & 1 & 27 & 90 \\
\hline Control group & 30 & 17 & 56.7 & 3 & 13.3 & 10 & 30 & 20 & 70 \\
\hline
\end{tabular}


version of the Chinese acne treatment guidelines, photodynamic therapy is recommended as an alternative treatment method for moderate-severe acne when systemic medication fails or is not tolerated by the patient. ${ }^{15}$ However, patients often experience intraoperative adverse reactions, such as erythema, edema, and pain, during the therapeutic operation. Studies show that pain during illumination is likely to be correlated with tissue injury, nerve stimulation, and differences in individual pain thresholds. ${ }^{16}$ Therefore, meticulous nursing is worthy of promotion in clinical practice for ensuring treatment effectiveness and safety.

Meticulous nursing includes aspects such as preoperative acne debridement, intra-operative nursing, postoperative skincare, pain care, daily care, psychological care, and lifestyle education. Zhang (a domestic researcher) et $\mathrm{al}^{17}$ used antimicrobial peptides combined with hyaluronic acid dressing to treat moderate acne vulgaris and found that it could effectively alleviate inflammatory skin lesions. In a study by $\mathrm{Xu},{ }^{18}$ a superficial dermal injury and skin barrier disruption mouse model was constructed after laser surgery, and it was confirmed that hyaluronic acid dressing could promote skin barrier recovery. Besides, immediate application of intermittent ice packs using the cold spray machine after treatment could not only treat the erythema but also reduce patient pain. In the present study, the cold spray machine process duration could be increased according to the specific patient's pain tolerance, and if necessary, music care could be added to reduce any negative emotions caused by the treatment, thus improving patient pain tolerance. ${ }^{19}$ Relevant studies show that, compared with patients with healthy skin, patients with acne have a more active sebaceous gland proliferation, with an increased sebum secretion and water loss through the epidermis as well as a water deficiency. Therefore, the use of topical anti-acne skincare products that control oil and repair the skin barrier is necessary in order to adjust the patient's skin condition and maintain the water-oil balance, thus stabilizing the effects of photodynamic therapy and preventing recurrence. ${ }^{20}$ Lifestyle education and guidance could help patients gradually develop healthy and regular lifestyle habits, thus helping them rebuild confidence and allowing the treated skin to recover. ${ }^{21,22}$ It is worth noting that related studies have shown a positive correlation between acne and the intake of whole, low-fat, and skimmed milk. Milk containing the bovine insulin-like growth factor (IGF-1), which binds to the human IGF-1 receptor and contains dihydrotestosterone precursors, including placental-derived progesterone, 5 $\alpha$-pregnenedione, and $5 \alpha$-androstenedione, may promote acne formation. ${ }^{23}$

The care process is carefully detailed and focuses on the disease while paying close attention to psychological care in the adolescent patients in order to improve treatment comfort and safety. Moderate-severe acne predominantly occurs in adolescent girls. As the majority of acne lesions are concentrated on the face, their presence may result in the disfigurement of facial appearance in severe cases. This can easily affect the patient's mood, cause depression or loneliness, and even lead to internet addiction. ${ }^{24}$ Therefore, this study conducted psychological care for the patients.

Therefore, in the present study, patients in the observation group were pretreated with acne debridement in a sterile operating room before photodynamic therapy. Acne squeezing can eliminate sebaceous duct blockage, ensure smooth sebum elimination, inhibit P. acnes reproduction, reduce inflammatory reactions, and accelerate the ALA absorption and penetration, thus improving treatment efficiency. After the 6-month follow-ups, which included the standardized 4 photodynamic treatments, it was observed that the acne lesions disappeared with satisfactory results. There was no recurrence, and no adverse reactions, such as keloids and hyperpigmentation, were observed. Patients should be instructed to continue with the routine daily care in the future in order to prevent recurrence.

In summary, the application mode of meticulous nursing in the photodynamic treatment of adolescent patients with moderate-severe acne might improve treatment safety and reduce postoperative adverse reaction occurrence. The method has good clinical effects and is worthy of further promotion.

\section{Ethics Approval and Consent to Participate}

This study was conducted with approval from the Ethics Committee of Beijing Hospital (No: 2021BJYYEC-16301). This study was conducted in accordance with the declaration of Helsinki. Written informed consent has been provided by the patient to have the case details and any accompanying images published.

\section{Consent for Publication}

All participants signed a document of informed consent. 


\section{Funding}

There is no funding to report.

\section{Disclosure}

The authors declare that they have no competing interests.

\section{References}

1. Lynn D, Umari T, Dunnick C, et al. The epidemiology of acne vulgaris in late adolescence. Adolesc Health Med Ther. 2016;7:13-25.

2. Zaenglein A, Pathy A, Schlosser B, et al. Guidelines of care for the management of acne vulgaris. $J$ Am Acad Dermatol. 2016;74:945-973.

3. Skin Laser Subspecialty Committee of Dermatologists Branch of Chinese Medical Association. [Consensus of 35 aminolevulinic acid photodynamic therapy for acne vulgaris]. Chinese Journal of Dermatology. 2011;44:75-76. Chinese.

4. Donnarumma M, Fattore D, Greco V, et al. How to increase adherence and compliance in acne treatment? A combined strategy of SMS and visual instruction leaflet. Dermatology. 2019;235(6):463-470. PMID: 31586999. doi:10.1159/000502575

5. Wu H, Xue M. [Photodynamic therapy combined with acne extrusion in the treatment of moderate to severe acne]. Chinese Journal of Aesthetic Medicine. 2018;27:39-42. Chinese.

6. Eberlein T, Wiegand C, Andriessen A, Roes C, Abel M. Use of a monofilament debridement pad in the treatment of acne vulgaris. $J$ Wound Care. 2019;28(11):780-783. PMID: 31721661. doi:10.12968/jowc.2019.28.11.780

7. Wu HE, Liu YB, Cui L, Xu GJ, Sun XD. Three-step irradiance schedule versus two-step irradiance schedule for pain control during topical 5-aminolevulinic acid-photodynamic therapy of facial acne in Chinese patients: a prospective randomized comparative study. Lasers Surg Med. 2021. PMID: 34368971. doi:10.1002/1sm.23464

8. National Guideline Alliance (UK). Skin Care Advice for People with Acne Vulgaris: Acne Vulgaris: Management: Evidence Review B. London: National Institute for Health and Care Excellence (NICE); June, 2021. PMID: 34424620.

9. Alexis AF, Woolery-Lloyd H, Williams K, et al. Racial/ethnic variations in acne: implications for treatment and skin care recommendations for acne patients with skin of color. J Drugs Dermatol. 2021;20 (7):716-725. PMID: 34232006. doi:10.36849/JDD.6169

10. Poli F, Dreno B, Verschoore M. An epidemiological study of acne in female adults: results of a survey conducted in France. $J$ Eur Acad Dermatol Venereol. 2001;15(6):541-545. PMID: 11843213. doi:10.1046/j.1468-3083.2001.00357.x

11. Kitaoka M, Nguyen TC, Goto M. Water-in-oil microemulsions composed of monoolein enhanced the transdermal delivery of nicotinamide. Int $J$ Cosmet Sci. 2021;43(3):302-310. PMID: 33566391. doi:10.1111/ics.12695
12. Wolkenstein P, Machovcová A, Szepietowski JC, Tennstedt D, Veraldi S, Delarue A. Acne prevalence and associations with lifestyle: a cross-sectional online survey of adolescents/young adults in 7 European countries. J Eur Acad Dermatol Venereol. 2018;32 (2):298-306. PMID: 28707712. doi:10.1111/jdv.14475

13. Shalita AR, Berson DS, Thiboutot DM, et al. Effects of tazarotene $0.1 \%$ cream in the treatment of facial acne vulgaris: pooled results from two multicenter, double-blind, randomized, vehicle-controlled, parallel-group trials. Clin Ther. 2004;26:1865-1873. doi:10.1016/j. clinthera.2004.11.012

14. Barbieri J, Spaccarelli N, Margolis J, et al. Approaches to limit systemic antibiotic use in acne: systemic alternatives, emerging topical therapies, dietary modification, and laser and light-based treatments. J Am Acad Dermatol. 2019;80(2):538-549. doi:10.1016/ j.jaad.2018.09.055

15. China acne treatment guidelines expert group. [China acne treatment guidelines (revised in 2019)]. Journal of Clinical Dermatology. 2019;48:583-588. Chinese.

16. Zheng Z, Zhang LL, Shi L, et al. What is the most relevent factor causing pain during ALA-PDT? A multi-center, open clinical pain score research trial of actinic keratosis, acne and condylomata acuminata. Photodiagnosis Photodyn Ther. 2019;26:73-78. doi:10.1016/ j.pdpdt.2019.03.001

17. Zhang RN, Xu W, Li Y, et al. [Clinical efficacy of antibacterial peptide combined with hyaluronic acid dressing in the treatment of moderate acne vulgaris]. Journal of Practical Dermatology. 2019;12:215-218. Chinese.

18. Xu LH, Gu H, Guo MH, et al. [Effect of hyaluronic acid on the repair of skin barrier function after laser injury in BALB/c mice]. Chinese Journal of Dermatology. 2014;47:345-348. Chinese.

19. Ma L, She LJ. [Current situation of nursing research on pain intervention with music in China]. Chinese Journal of Nursing. 2008:268-271. Chinese.

20. He L, Hao F. [Application guide of anti acne skin care products in acne].The Chinese Journal of Dermatovenereology. 2019;33:1107-1109. Chinese.

21. Fan H, Tao SQ; In Chinese. [Clinical observation and nursing of the treatment of moderate and severe acne with minimally invasive combined with photodynamic therapy]. International Medicine and Health Guidance News. 2017;23:2934-2937.

22. Wang F, Liu YB, Sun WY. [Comprehensive nursing experience of photodynamic therapy for moderate and severe acne]. China Medical Cosmetology. 2018;8:78-81. Chinese.

23. Aghasi M, Golzarand M, Shab-Bida S, et al. Dairy intake and acne development: a meta-analysis of observational studies. Clin Nutr. 2019;38(3):1067-1075. doi:10.1016/j.clnu.2018.04.015

24. Öztekin C, Öztekin A. The association of depression, loneliness and internet addiction levels in patients with acne vulgaris. Biopsychosoc Med. 2020;14:17. doi:10.1186/s13030-020-00190-y
Clinical, Cosmetic and Investigational Dermatology is an international, peer-reviewed, open access, online journal that focuses on the latest clinical and experimental research in all aspects of skin disease and cosmetic interventions. This journal is indexed on CAS.
The manuscript management system is completely online and includes a very quick and fair peer-review system, which is all easy to use. Visit http://www.dovepress.com/testimonials.php to read real quotes from published authors. 